\title{
Smart halal recognizer for muslim consumers
}

\author{
Siti Fatimah Abdul Razak, Chin Poo Lee, Kian Ming Lim, Pei Xin Tee
}

Faculty of Information Science and Technology, Multimedia University, Malaysia

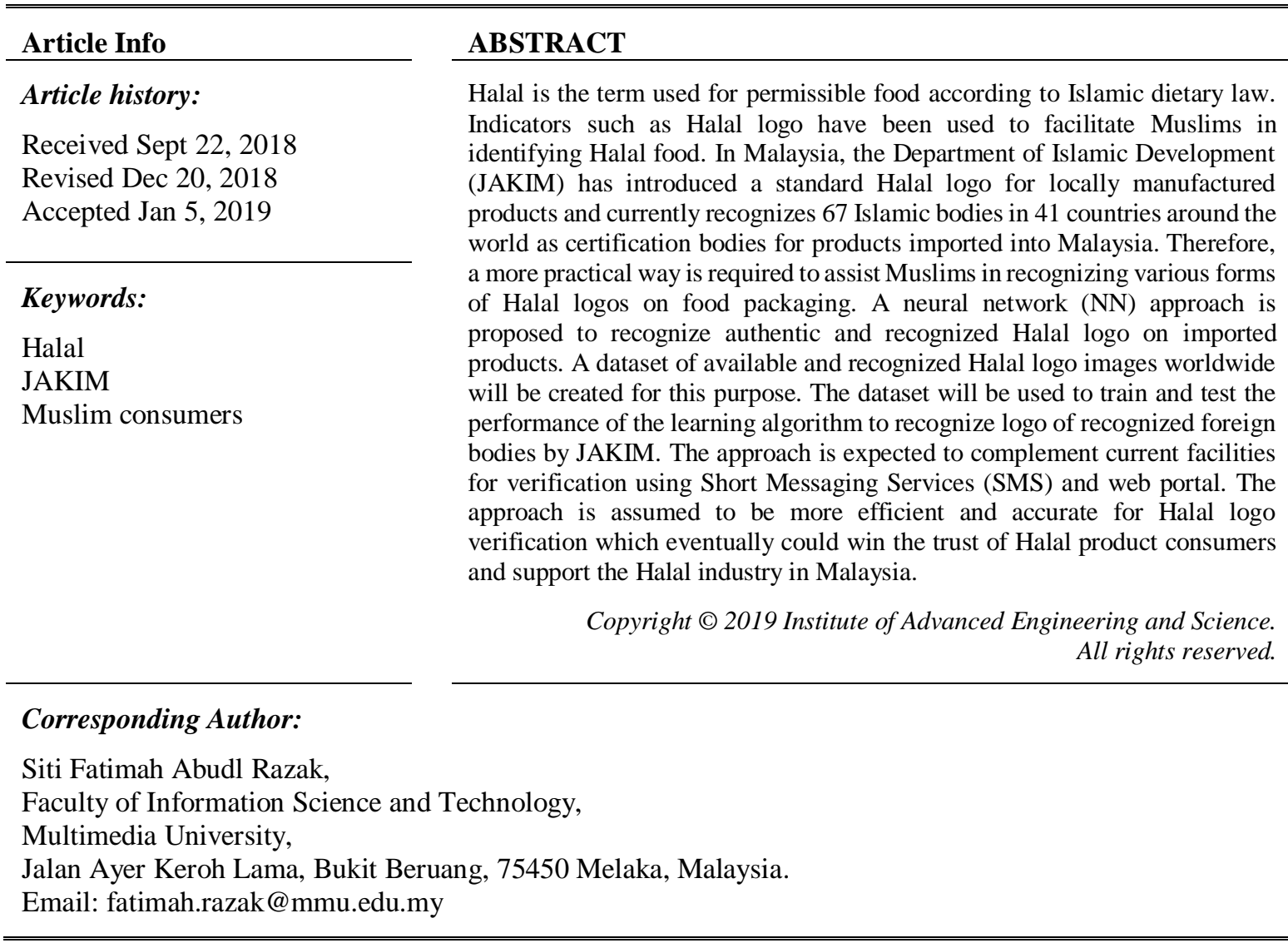

\section{INTRODUCTION}

Muslims are only permissible to consume food which are Halal. In order to recognize this group of food from the market, they normally depend on the printed Halal logo which can be found on food packaging. They assume that if a Halal logo is visible on the packaging, then the food is permissible to be consumed. However, a standardized and globally used Halal logo is yet to be available. Therefore, the logo depends on individual provinces or states or non-governmental organizations (NGOs) which issued the Halal certificate based on manufacturers applications. As one of the main player in the global Halal industry, Malaysia implements a standardized Halal logo issued by the Department of Islamic Development (JAKIM). Local manufacturers are required to apply to JAKIM and go through a thorough background check before a manufacturer is certified and allowed to display the Halal logo. In addition, JAKIM currently recognizes 67 Islamic bodies in 41 countries around the world as a reputable and credible foreign halal certification bodies as JAKIM representatives to monitor and verify the halal status of imported products. Imported products have various Halal logo printed on the packaging which depends on the issuer. Therefore, to memorize and recognize these Halal logos are very challenging. Muslim consumers who face doubts in this matter will call the authorities to raise their concerns and confirm the validity of the Halal status of the product. Else, they will abandon the intention to purchase the product.

JAKIM realizes the challenges faced by general Muslim consumers in Malaysia. Therefore, JAKIM has provided a Short Messaging Service (SMS), termed "SMS Halal JAKIM". This service allows consumers to send text message, i.e. HALAL SEMAK <company registration number> to 15888 for Halal checking 
purpose. However, consumers need to wait for a reply which may be time consuming. In addition, a web portal is also accessible for consumers to perform self-check on a product's Halal status by providing information like product brand, product name and manufacturer name. A list of recognized foreign bodies is also available in .pdf format for consumers to verify Halal status for imported food products. In practical, Muslim consumers face difficulties to recognize the logo of international certification bodies on-the-go. The tasks of recognizing certified foreign Halal logo is generally challenging and referring to a dedicated list before purchasing an imported product is not very practical.

Therefore, this paper presents an implementation of neural network to recognize authentic and JAKIM's recognized Halal logo images. The paper is organized as follows: Section I introduces the subject matter, Section II presents literature review which include the available Halal technologies and related work in Malaysia Halal Industry, Section III highlights the methodology and explained the process. Section IV presents the results and analysis of the neural network performance. The last section is Section V which concludes this paper.

\section{LITERATURE REVIEW}

Halal logo printed on food packaging have been an acceptable indicator for Muslims to recognize permissible food according to the religion. Their intention to purchase a certain type of food is positively influenced by their Halal awareness and certification [1]. Products with Halal logo attracts more consumers in countries with majority are Muslims such as Malaysia. Hence, there have been attempts to assist consumer in recognizing the Halal products using information technology especially in Malaysia [2] as summarized in Table 1, Table 2 and Table 3.

Table 1 summarizes machine-based Halal technologies which have been introduced in Malaysia. The implementation of these technologies can be found in selected hypermarkets. Table 2 on the other hand, summarizes web-based technologies which are accessible by Muslim consumers 24/7 using various devices with Internet access. Lastly, Table 3 provides a summary of mobile-based Halal technologies which have been developed for mobile users.

Table 1. Machine-Based Halal Technologies

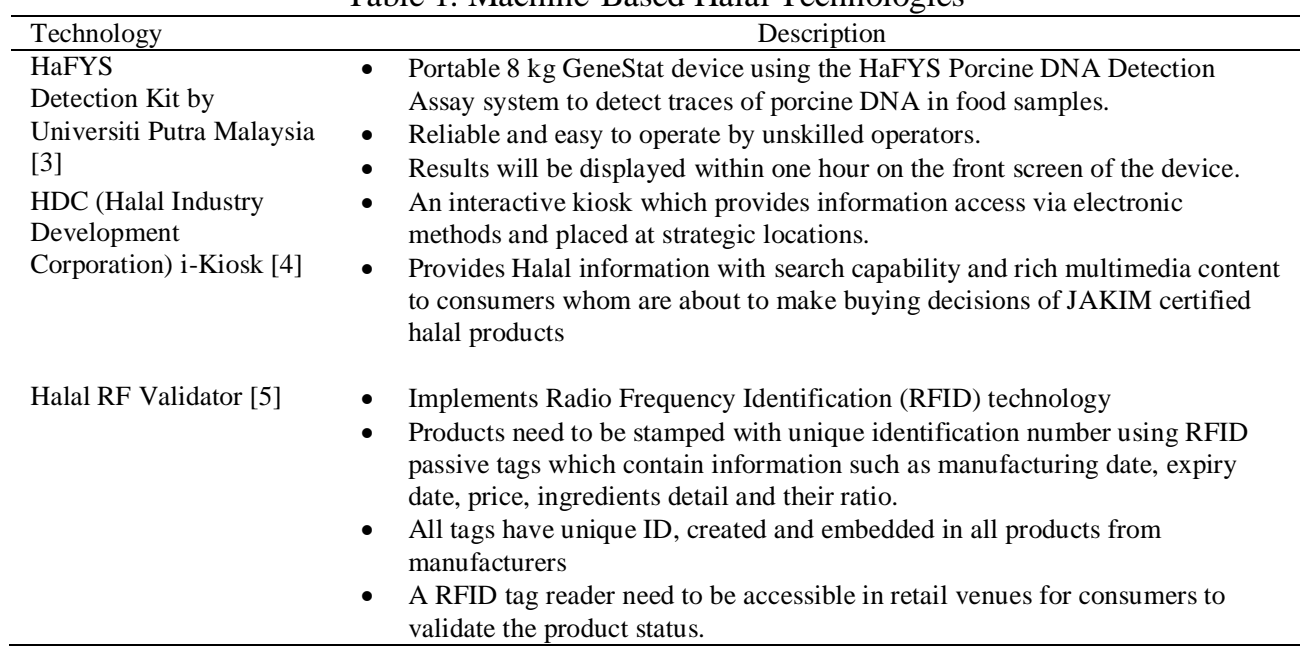

Table 2. Web-based Halal Technologies

\begin{tabular}{lll}
\hline \multicolumn{1}{c}{ Technology } & & \multicolumn{1}{c}{ Description } \\
\hline MYeHALAL [2] & - & Support Halal certification application system for domestic and \\
& & international applicants \\
& - Available in English and Malay language only \\
Halal Malaysia & - & Provide database search function based on states, categories and \\
Directory [5] & & keywords. \\
& - & Search results are displayed in a table listing. \\
& - Information on product manufacturers, Halal status validity and \\
& & conferring authorities are available. \\
HDC Halal Widget & - Internet access is required. \\
[6] & Provide information based on the Department of Islamic \\
& & Development Malaysia (JAKIM)'s certified products.
\end{tabular}




\begin{tabular}{ll}
\hline \multicolumn{1}{c}{ Technology } & \multicolumn{1}{c}{ Description } \\
\hline & - Bloggers and website owner can place the widget on their website. \\
& $-\quad$ Provide widgets to be displayed on the HDC website. \\
& The product dropdown list is not available. The user has to enter it \\
& Limitation only given to the industry size and state only. No \\
& limitations of displayed information at one time. \\
& More time needed for the search process as the user needs to think \\
& and enter keyword before searching \\
Halal Ingredients & Companies need to key in the category, source and status of their \\
Database & product ingredients. \\
{$[2]$} & User can search for status of E-numbers
\end{tabular}

MyMobi

Halal 2.0 [8]

HDC Halal Mobile Apps [9]

HDC Nokia Apps [10]

\author{
HDC \\ iPhone [11] \\ Verify Halal [12]
}

Smart Halal App [13]

MyHALAL2U [14]

Halal-Square [15]

Halal Finder [16]

Table 3. Mobile-based Halal Technologies

- Specially design for consumers to validate Halal status on food products by sending 13-digit barcode number to JAKIM

- Limited to plaintext representation only.

- Time consuming and prone to typographical errors

- Requires consumers to have a mobile phone and enough credit balance to send a text message.

- Mobile phone providers will charge USD0.10 (MYR0.35) cents per SMS to use the SMS Halal JAKIM

- Using Multimedia Messaging Service (MMS) to validate product status

- Send barcode image (minimum one-megapixel camera is required) using mobile phone for validation.

- Higher cost compared to SMS.

- Locate Halal premises location within $20 \mathrm{~km}$ radius from the current location Use Global Positioning System (GPS) technology and Google Maps.

- Additional features include cleanliness and hospitality ratings, mark favourite premises, social media integration, support e-mail and call integration

- Link to Halal Malaysia Directory

- Locate Halal premises location within $15 \mathrm{~km}$ radius from the current location Use Global Positioning System (GPS) technology and Google Maps

- Can access the validity of the Halal status of products, premises and abattoirs.

- Similar to HDC Halal Mobile Apps but limited for iPhone, iPod Touch and iPad users only.

- Directly connected to JAKIM's database

- Consumers and retailers can instantly check the halal integrity of any product.

- Easier for users to receive fast and accurate information related to verify Malaysian halal certification

- Enable consumers to check the status of food \& beverage products, food premises, slaughterhouses, consumables, cosmetics \& pharmaceutical products and halal-certified logistics services by the Malaysian Halal Certification.

- Include bar code and QR code scanner

- Similar to Smart Halal App

- NFC-based app to verify the authenticity of the Halal Certificate

- Fully integrated with Halal-Inside.com for information sharing purpose via reviews

- Location based notification to nearby Halal restaurants with search (serial number, premise name) and direction (Google Street View) functions

- Additional features include special promotions opportunities.

- An application to identify the packaging of non-halal and halal food.

- Can search Halal additives and Halal logo.

- Limited descriptions

Available Halal technologies have their own advantages and disadvantages. In addition, the process of recognizing and dividing free-form graphical arrangement like logos is quite demanding. Variations in logo 
style and image quality makes the process even more challenging [17]. Hence, previous researches have proposed the application of learning algorithms to facilitate the process. The most recent is a neural radio frequency identification (neural-RFID) based model which assists consumers to determine Halal and non-Halal food products. The RFID reader reads all basic information from RFID tags and feeds to the neural network, already trained using back propagation learning algorithm. The trained neural network immediately votes for halal or non-halal [18].

Prior to that, [5] developed an automatic Halal product identification using passive RFID tags. These tags can be simply scanned to validate the Halal status of products. Profiles of products are prepared based on similar database of the Islamic authority in Malaysia.

In another work, [19] developed a four-phased authorized JAKIM's Halal logo detection and recognition system The phases are based on four modules which are image acquisition, pre-processing, detection and recognition module. The image recognition module implements neural network approach to recognize whether the Halal logo is authorized or not.

\section{METHODOLOGY}

\subsection{Research Methodology}

This research was completed within eighteen months, from June 2017 until February 2018. It involves a few phases i.e. review and analysis, design, prototyping, evaluation and testing. In the beginning of the research, available and existing technologies as well as approaches in validating Halal status of products were reviewed. In order to gain in-depth understanding related to the subject matter, electronic documents from several electronic databases such as ISI, SCOPUS and ACM were also studied. The findings and results of this phase are summarized in Table 1, Table 2 and Table 3.

A process model as shown in Figure 1 was developed in the next phase. Then, a self-collected Halal logo images dataset was created. A prototype was developed using MATLAB. System tests were conducted after implementation to ensure that the prototype is able to recognize the Halal logo images accurately.

In this section, it is explained the results of research and at the same time is given the comprehensive discussion. Results can be presented in figures, graphs, tables and others that make the reader understand easily [2], [5]. The discussion can be made in several sub-chapters.

\subsection{System Architecture}

The structure, behavior and view of the system is illustrated using a conceptual model as in Figure 1. The system architecture is a conceptual model that defines the structure, behavior, and more views of the system. The architectural description is a systematic description and representation of the organization in order to support reasoning about the structure and behavior of the system. The ability of systems to identify or recognize objects basically known as machine vision. The computer can use machine vision technology and artificial intelligence algorithms to achieve recognition. The system loads the image and processes the logo images, the learning algorithm learns the logo images and searches from the training dataset and produces an outcome. We adopted the logo identification and recognition system architecture [20] and applied the fundamental processes in computer vision and image recognition which includes image acquisition, preprocessing and classification, feature extraction, recognition and output or result.

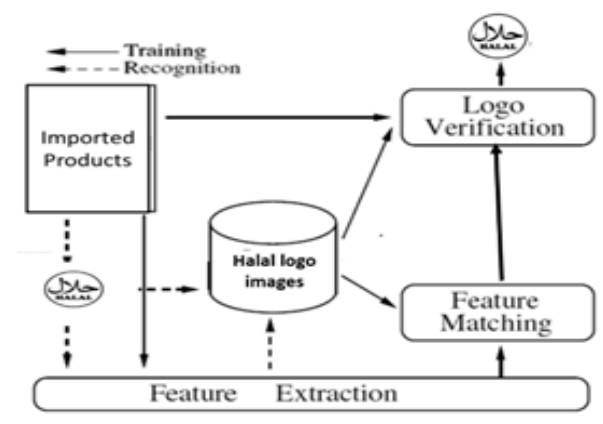

Figure 1. System architecture (adopted from [20])

The image acquisition process is the first step in any object recognition system. In this work, the dataset is a Halal logo image dataset. All 920 logo images were captured directly from products packaging 
using a mobile phone. The acquired images were obtained from different angles and light conditions before used to train the learning algorithm. This is important to allow the learning algorithm to learn a variety of visual appearances which may contribute to higher recognition accuracy. Examples of the obtained logo images are shown in Figure 2.

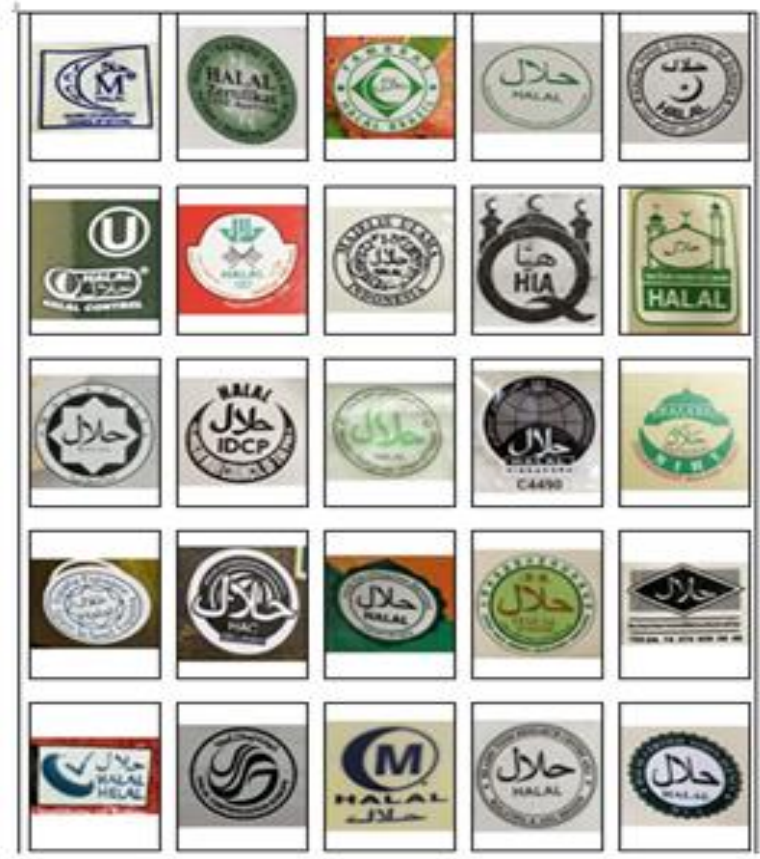

Figure 2. Examples of Acquired Halal logo

The acquired images were grouped into ten different folders, based on the origin of the images (Country) as documented in JAKIM's .pdf file of International Foreign Certified Bodies, which were downloaded from JAKIM's official Halal portal. The images of each of the ten countries were cropped to represent reference images of each country, which were later used for recognition purpose. Summary of the number of images according to the country is shown in Table 4.

Table 4. Classification of Halal Logo Based on Country

\begin{tabular}{lc}
\hline \multicolumn{1}{c}{ Country } & Number of images \\
\hline Australia & 50 \\
Austria & 5 \\
China & 119 \\
Europe & 52 \\
Indonesia & 117 \\
Italy & 53 \\
Korea & 37 \\
Malaysia & 376 \\
Philippine & 44 \\
Singapore & 67 \\
\hline
\end{tabular}

Since the images were directly acquired from food packaging, they vary in terms of layout and size. The images were pre-processed in a manner that accelerates the performance of other processes afterwards. The images were resized to ease the feature extraction process. The quality of the images may also be improved through contrast enhancement, noise reduction and image restoration. In addition, the images may also be compressed to reduce the storage space or bandwidth required for transmission. The images from each folder were loaded by categories before resizing to $64 \times 64$ dimensions. There were also different kinds of orientation of the images. Some images are taken from above and some are from the sides. The diversity of appearances of the images in each type of logo made it difficult to choose the best features for the system to differentiate between categories. 
The Halal logo images were then fed into neural network. Neural network is an effective and efficient platform theory to predict and know a situation if appropriate model design architecture and input data were available. The learning algorithm can be trained to classify images according to their categories in order to more accurate results. However, a large amount of input data needs to be input.

The back propagation neural network was used to identify the Halal logo images. Neural Network Toolbox ${ }^{\text {TM }}$ provides pattern recognition and classification features such as nprtool, which is a neural network pattern recognition tool available in MATLAB. It uses a two-layer feed-forward pattern recognition network with S-shaped output neurons. The nprtool function was implemented to train, validate and test the created network in one run so the distribution of the dataset was set before the training begins. By default, the training dataset was set to $90 \%$, validation dataset was set to $5 \%$ and testing dataset was set to $5 \%$. The input vectors and target vectors were randomly divided into these three sets. For this system, several patterns of setting had been experimented. It was discovered that the default setting is one of the best, thus it was chosen. The number of hidden neurons was also set to default i.e. ten hidden neurons in the hidden layer. The training was then performed.

\section{RESULTS AND ANALYSIS}

The performance of the learning algorithm i.e. neural network was evaluated using confusion matrix and Receiver Operating Characteristics (ROC).

The confusion matrix was plotted to analyze the accuracy and the error rate for the trained network. Figure 3 shows the confusion matrix for the trained neural network. The green highlighted boxes show the correct matches of the output and target class while the red highlighted boxes show the wrong matches. The accumulative percentage of right and wrong matches was stated in the blue highlighted box.

Based on Figure 3, the accuracy rate during training is quite high which is $95.7 \%$. Since this is the best accuracy rate of the trained neural network through several repetitions of the training process, it was very promising. The training images were fed into the system one-by-one and the recognition results were recorded. The trained neural network is then saved and a command line script is generated at the end. The trained neural network was later used to classify the input image from the system. During validation, the accuracy is $72.5 \%$. Testing was performed to evaluate the of the recognition module and recorded a recognition rate of $72.5 \%$. This was done using a dataset of five test images per category of the Halal logo images. These test images are made sure that they are not the same as the training images used for training. Overall, the accuracy rate of the trained neural network is $88.7 \%$.

Additionally, Figure 4 shows the performance of the neural network based on the ROC. The trained $\mathrm{NN}$ was loaded into the system and used to classify the image inserted by the user and the output will be a vector of ten different values (for ten categories). The highest value shows the high confidence of the NN that the image belongs to that certain category.
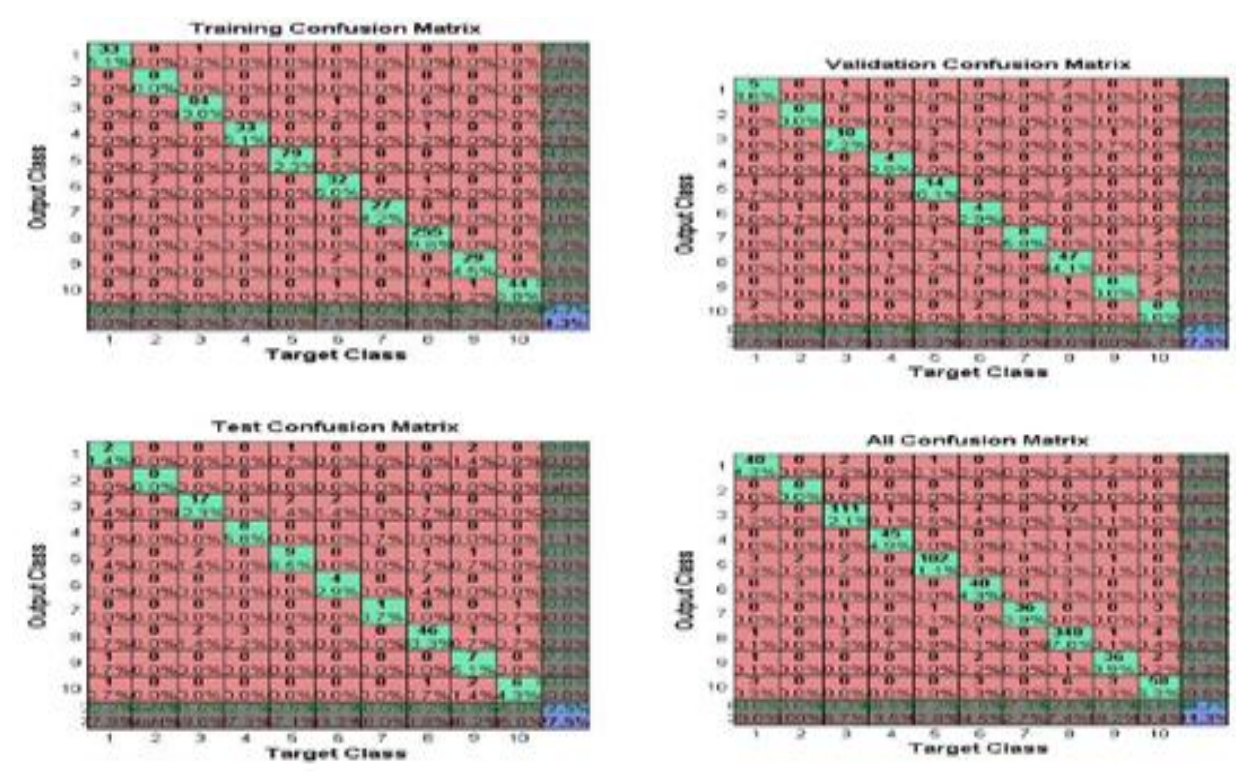

Figure 3. Confusion Matrix for training, testing, validation and overall performance of trained neural network 

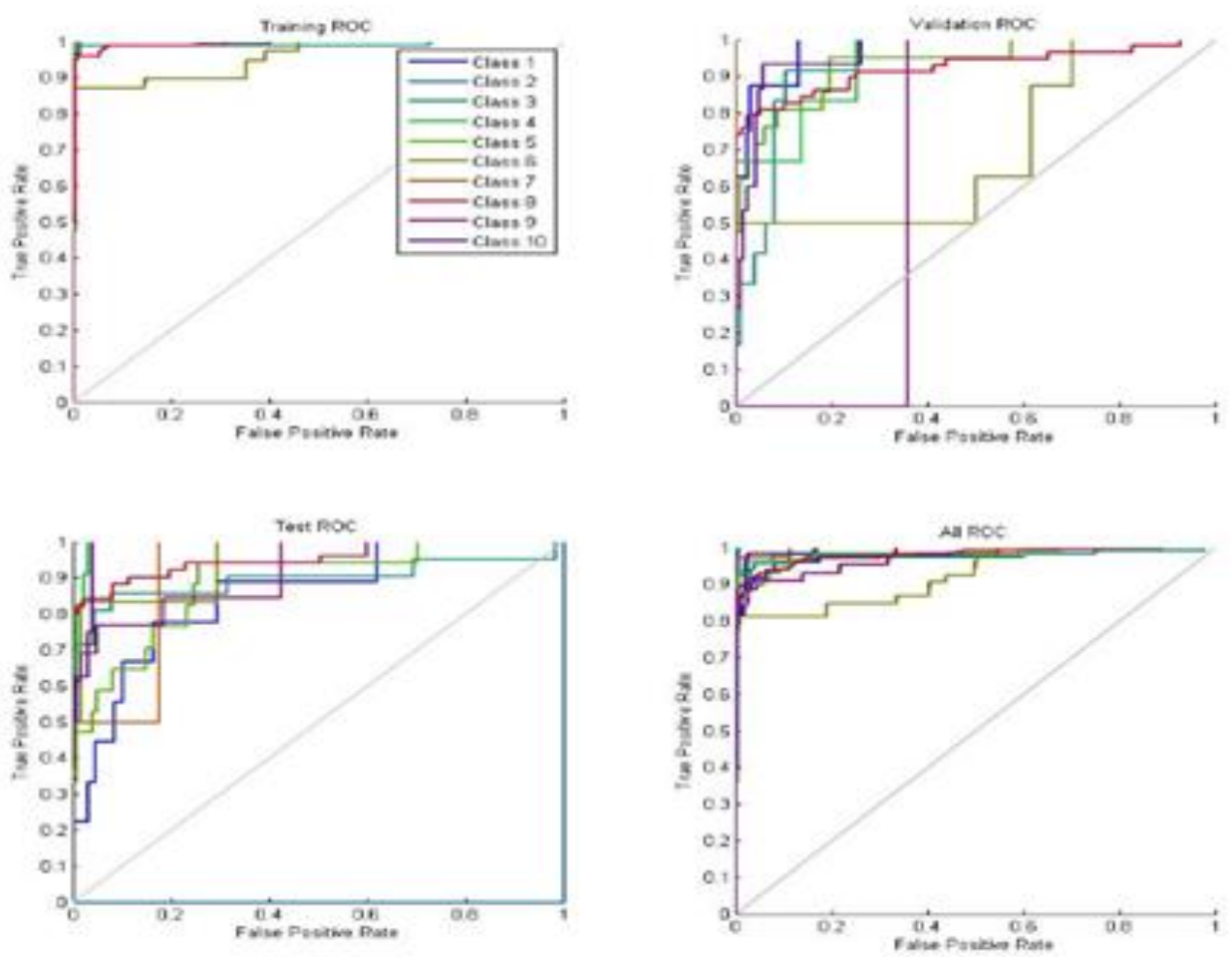

Figure 4. ROC of Trained Neural Network

\section{CONCLUSION}

Even though there are a number of available Halal technologies available to facilitate Muslim consumers in Malaysia to authenticate the Halal logo images, previous work focused on recognizing JAKIM's Halal logo. No special attention was given to Halal images of certified foreign international bodies. Thus, we intend to fill up this gap. We explored the potential of neural network to recognize these logo images. We created a dataset of these images which were self-captured using built-in mobile phone camera. We trained and tested the performance of neural network to identify and recognize Halal logo images from ten different countries. Results may serve as a benchmark for the authorities to provide a comprehensive database which enable consumers to get prompt results before committing to any purchases especially imported products. We plan to extend this work to include Halal logo images from all 64 authorized and certified foreign bodies and allow real time recognition process using mobile app.

\section{REFERENCES}

[1] Y. A. Aziz and C. N. Vui, C.N, "The Role of Halal Awareness and Halal Certification in Influencing Non-Muslim's Purchase Intention," 3rd International Conference on Business and Economic Research, 2012, Bandung, Indonesia, paper 1822.

[2] Z. J. M. Husny, M. I. I. Tan, I. S. Ibrahim, I. S. and M. Z. A. Rozan, “Assessment on the Utilization of Current Halal Certification Technologies by Halal Industry Players in Malaysia," in Proceeding of The World Congress on Engineering and Computer Science, 2014, vol. 2, pp. 22-24.

[3] L. K. Shagar, "Portable device can test for porcine DNA within an hour", The Star Online, 31 March 2016. Retrieved from https://www.thestar.com.my/news/nation/2016/03/31/portable-device-can-test-for-porcine-dna-within-anhour/\#juGpRuX1ygIWk1x5.99

[4] HDC, "HDC i-Kiosk". Retrieved from http://www.hdcglobal.com/publisher/ci hdc i kiosks

[5] Nasir, Mohd, Azah Norman, Shukor Sanim Bin Mohd Fauzi, and Masliyana Azmi. "An RFID-based validation system for halal food." Int. Arab J. Inf. Technol. 8, no. 2 (2011): 204-211.

[6] HDC, "Introducing the World's First Halal Directory Widget," HDC VIBE 2009, 19.

[7] JAKIM, "SMS: Facility checking application status," 2010, Retrieved from. http://www.halal.gov.my/v2/index.php?ty=content_view\&id=ANN-20100503105536\&type=ANN

[8] S. N. Junaini and J. Abdullah, "MyMobiHalal 2.0: Malaysian Mobile Halal Product Verification using Camera Phone Barcode Scanning and MMS," in Proceedings of the International Conference on Computer and Communication Engineering 2008, Kuala Lumpur, Malaysia. 
[9] Halal Industry Development Corporation - Halal Directory, (2013), Retrieved from http://www.hdcglobal.com/publisher/alias/bu_halal_directory?dt.driverAction=RENDER\&pc.portletMode=view\& pc. windowState=normal \&pc.portletId=Hadir-web.. HadirPortlet

[10] HDC, "HDC Nokia Application," 2011, Retrieved from http://www.hdcglobal.com/publisher/cu_hdc_nokia_application

[11] HDC, "Halal App Now Available for iPhone," 2010. Retrieved from http://www.hdcglobal.com/publisher/bu_hdc_iphone_app

[12] Serunai, "Global Halal Data Pool,” 2016, Retrieved from http://globalhalaldatapool.com:8383/Home/Info

[13] JAKIM and MCMC, "Smart Halal App", 2017, Retrieved from https://play.google.com/store/apps/details?id=my.gov.islam.smarthalal\&hl=en

[14] JAKIM, "MyHALAL2U", 2017, Retrieved from https://play.google.com/store/apps/details?id=my.halal.jakim\&hl=en

[15] "Halal Square", 2015, Retrieved from https://play.google.com/store/apps/details?id=com.myms.halalsquare\&hl=en

[16] "Halal Finder", 2015, Retrieved from https://play.google.com/store/apps/details?id=com.halalfinderapp\&hl=en

[17] G. Zhu and D. Dorman, "Logo matching for document image retrieval," in 10th International Conference on Document Analysis and Recognition 2009, pp. 606-610. IEEE.

[18] T. Saba, "Halal food identification with neural assisted enhanced RFID antenna," Biomedical Research 28, no. 18, 2017.

[19] Mohd, Hj, Mohd Norzali, Abd Wahab, Mohd Helmy, and Amir Yaakob. "Halal logo detection and recognition system," in Proceedings of the 4th International Conference of Information Technology and Multimedia (ICIMU' 2008), 17th - 19th November 2008.

[20] Z. Li, M. S. Austum and M. Neschen, "Fast Logo Detection and Recognition in Document Images," 2010 International Conference on Pattern Recognition, 2010, pp. 2716-2719.

\section{BIOGRAPHIES OF AUTHORS}

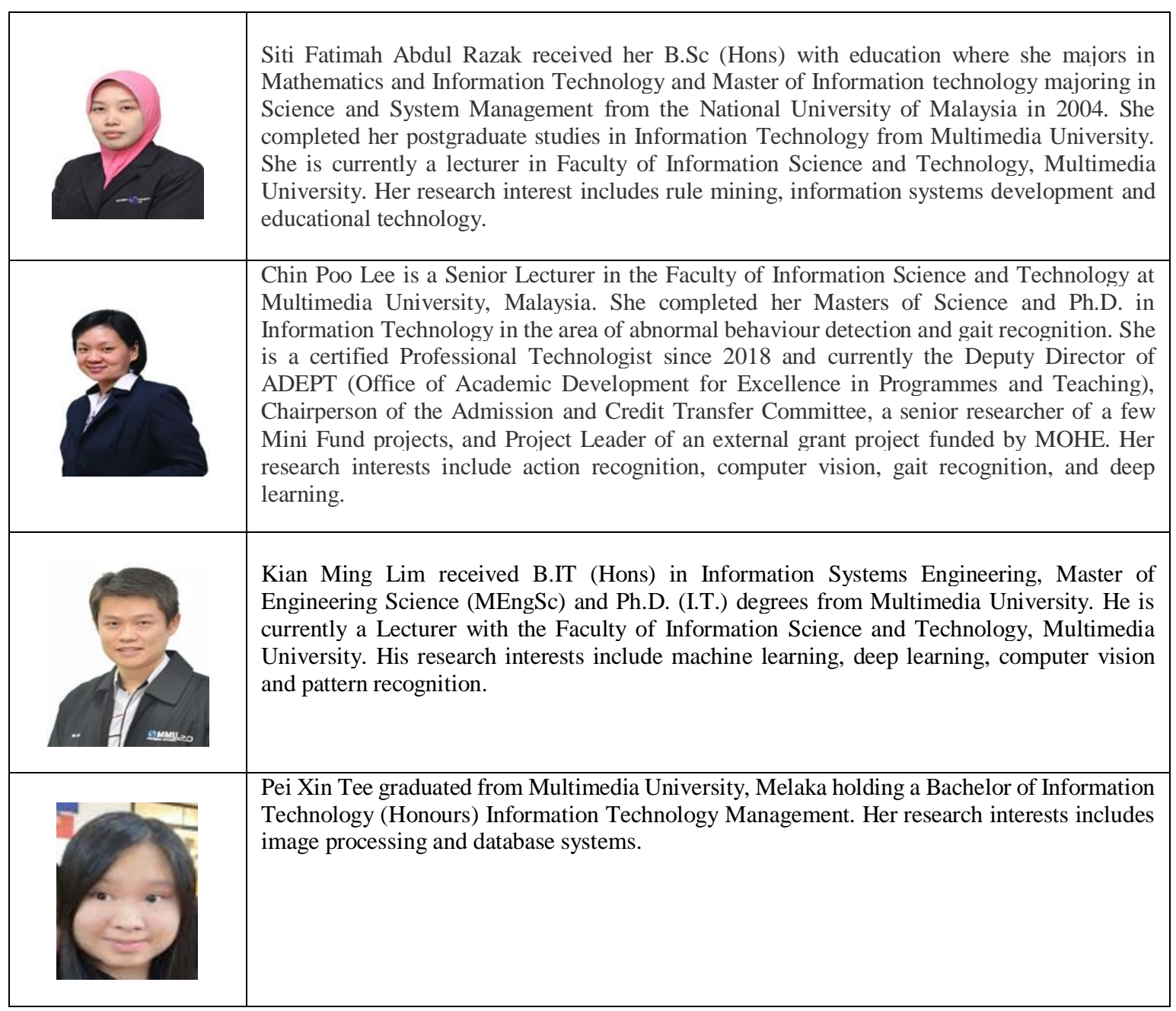

